\title{
Management of respiratory failure in patients with the acquired immune deficiency syndrome and Pneumocystis carinii pneumonia
}

\author{
Robert F Miller, David M Mitchell
}

Pneumocystis carinii pneumonia is the most common life threatening infection in patients with AIDS and is responsible for up to $85 \%$ of all respiratory episodes. ${ }^{1}$ The disease ranges in severity from a mild infection with a normal chest radiograph and an indolent course $\mathrm{e}^{2}$ to respiratory failure requiring assisted ventilation, where the mortality may exceed $90 \%{ }^{3}$ Pneumocystis pneumonia is now likely to be diagnosed at an earlier stage than it was in the past because of increased awareness by both physicians and patients and also because of the increased sensitivity of the bronchoscopic and laboratory methods now available. Despite these diagnostic advances and increased awareness, the mortality of pneumocystis pneumonia has not fallen. ${ }^{3}$ Several recent studies have attempted to identify which patients with pneumocystis pneumonia are likely to have a poor outcome. ${ }^{45}$ These studies have looked at several aspects, including patterns of symptoms and clinical findings, the degree of abnormality on the chest radiograph, measures of oxygenation and results of laboratory tests such as serum lactate dehydrogenase activity. The prognostic markers associated with a poor outcome are summarised in table 1 . In essence, these studies show that patients with severe pneumocystis pneumonia do badly and that there is no reliable index of prediction that will permit clinicians to identify prospectively those patients who are likely to have a poor disease course. This article will discuss the management of HIV positive patients with pneumocystis pneumonia who deteriorate while having treatment and develop respiratory failure.

\section{Therapeutic options for deteriorating pneumocystis pneumonia}

University College and Middlesex School of Medicine, Middlesex Hospital, London R F Miller

St Mary's Hospital, London

D M Mitchell

Address for reprint requests: Dr R F Miller, Department of Medicine, UCMSM, Middlesex Hospital, London WIN 8AA. to $85 \%$ of patients admitted pneumocystis pneumonia will respond to intravenous pentamidine or high dose co-trimoxazole. ${ }^{6}$ Patients will normally improve by the fifth day of treatment, somewhat more slowly than with bacterial pneumonia. Defervescence of fever, improved arterial blood gas tensions, and reduction of dyspnoea will have occurred in most patients by the seventh day of treatment; improvements in the chest radiograph are often delayed and may take two or three weeks. A few patients will deteriorate despite treatment. When this happens the diagnosis should be reviewed and further investigations considered (table 2). Several distinct patterns of deterioration are seen in pneumocystis pneumonia (table 3 ). Faced with a patient who is deteriorating despite conventional treatment or who is severely ill on admission the clinician has various therapeutic options once he has excluded alternative diagnoses or identified infection with $P$ carinii with or without a copathogen and begun appropriate treatment (table 4).

Changing to intravenous co-trimoxazole Some patients with mild to moderate (non-hypoxaemic) pneumocystis pneumonia treated with nebulised pentamidine will develop hypoxaemia, due possibly to the physicochemical properties of the drug. ${ }^{7}$ The hypoxaemia should be corrected with supplemental oxygen and treatment switched to intravenous high dose co-trimoxazole.

Patients already having intravenous co-trimoxazole Up to $90 \%$ of patients who fail to respond to intravenous co-trimoxazole will fail to respond to intravenous pentamidine; changing from one drug to the other may not be of benefit. ${ }^{8}$ Both drugs have slow mean response times of four to seven days. ${ }^{6}$ Pentamidine in particular seems to be associated with a slow response with a delay in improvement in oxy-

Table 1 Predictors of a poor outcome from Pneumocystis carinii pneumonia

Prolonged history of dyspnoea/dry cough ( $\geqslant 4$ weeks)

Admission respiratory rate $>30 / \mathrm{min}$

Recurrent Pneumocystis carinii pneumonia (second, third, or fourth episode)

Copathogens in bronchoalveolar lavage fluid

Poor oxygenation at admission: $\mathrm{PaO}_{2}<53 \mathrm{~mm} \mathrm{Hg}(7.0 \mathrm{kPa})$

Alveolar-arterial oxygen gradient $>30 \mathrm{~mm} \mathrm{Hg}(4.0 \mathrm{kPa})$ serum albumin at admission $(<35 \mathrm{~g} / \mathrm{l})$

Severe radiographic abnormalities ("white out")-that is diffuse bilateral interstitial infiltrates with or without alveolar consolidation

Raised white blood cell count at admission ( $\left.>10.8 \times 10^{9} / 1\right)$

Substantially increased serum lactate dehydrogenase activity ( $>300 \mathrm{IU} / \mathbf{1})$

Severe interstitial oedema in transbronchial biopsy specimens (a measure of the severity of diffuse alveolar damage) 
Table 2 Causes of deterioration in patients with Pneumocystis carinii pneumonia

Severe, progressive pneumocystis pneumonia

Severe drug side effects

Wrong diagnosis (for example, really staphylococcal pneumonia or intrapulmonary Kaposi's sarcoma)

Copathogen infection (for example, mycobacterial infection as well as pneumocystis pneumonia)

Other complications:

pneumothorax

pneumothorax
left ventricular failure

anaemia

genation (as measured by the alveolar-arterial oxygen gradient. $)^{6}$ This may be due to slow intrapulmonary accumulation of pentamidine when this is given by the intravenous route. 9

SALVAGE TREATMENT FOR PATIENTS FAILING TO RESPOND TO INITIAL TREATMENT

Trimetrexate (a methotrexate analogue) is a potent inhibitor of the dihydrofolate reductase enzyme of $P$ carinii and because it is lipid soluble it readily penetrates the organism. Its use is limited by myelosuppression. As $P$ carinii lacks a folate transport system, however, this provides the opportunity for differential rescue of host tissues with folinic acid without reversal of the antiprotozoal effects. In a study of 16 patients with AIDS and pneumocystis pneumonia who failed to respond to both pentamidine and co-trimoxazole (or were intolerant of them) and were therefore given trimetrexate $\left(30 \mathrm{mg} / \mathrm{m}^{2} /\right.$ day) and folinic acid $\left(80 \mathrm{mg} / \mathrm{m}^{2} /\right.$ day $), 11$ responded and 11 survived. A further 16 patients who were intolerant of cotrimoxazole received trimetrexate, of whom 10 responded and 13 survived. ${ }^{11}$ The use of trimetrexate was complicated by neutropenia, abnormal liver function and rash. There was also a high incidence of relapse after successful treatment with trimetrexate.

Eflornithine, an inhibitor of protozoal ornithine decarboxylase, has also been used in salvage studies of patients with pneumocystis infection who failed to respond to initial treatment. ${ }^{11}$ In the largest study 345 patients with pneumocystis pneumonia who were initial treatment failures, or who were intolerant of co-trimoxazole or pentamidine or both, received eflornithine $400 \mathrm{mg} / \mathrm{kg}$ a day intravenously. ${ }^{12}$ Of those patients who received assisted ventilation when eflornithine was started and who completed 14 days' treatment, $23 \%$ survived; of those who were breathing spontaneously at the start of treatment, $78 \%$ survived. Evaluating the results of such studies in patients whose survival rate may be poor anyway is difficult but our experience suggests that up to $60 \%$ of

Table 3 Patterns of deterioration in Pneumocystis carinii pneumonia

\footnotetext{
Rapid deterioration over $24-48$ hours after admission

Initial mild disease at admission with response to treatmen and sudden deterioration at day 5 ("five day dipper")

Slow downwards grumbling deterioration over 7-15 days

Sudden deterioration over hours or minutes at any stage: exclude pneumothorax left ventricular failure

Deterioration after fibreoptic bronchoscopy and lavage
}

Table 4 Therapeutic options in a deteriorating patient with Pneumocystis carinii pneumonia

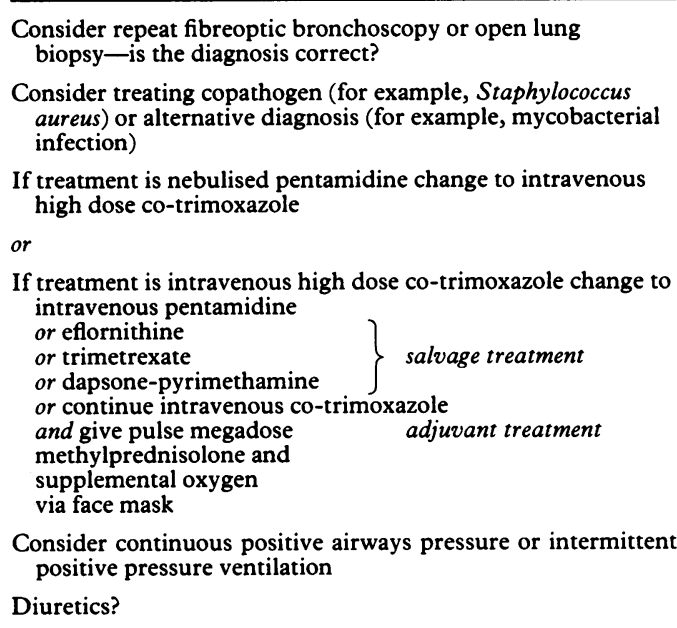

patients who have deteriorated or failed to respond to high dose co-trimoxazole or pentamidine will show a response to eflornithine. ${ }^{13}$ Toxic effects include blood dyscrasias (usually thrombocytopenia) in up to half of the cases, and diarrhoea is also seen.

\section{ADJUVANT TREATMENT}

Methylprednisolone

The evidence for benefit from corticosteroid treatment is based on anecdotal reports and open prospective trials. ${ }^{14} 15$ How glucocorticoids might promote resolution of pneumocystis pneumonia is unknown. Steroid induced reduction of chemotaxis and phagocytic activity of inflammatory cells and reduction in complement mediated neutrophil activation may protect against inflammatory lung damage. ${ }^{16}$ The overwhelming inflammatory response to severe pneumocystis infection may result in pulmonary damage and rapid clinical deterioration, fulfilling the criteria for a diagnosis of the adult respiratory distress syndrome. ${ }^{17}$ Given the slow response time for intravenous co-trimoxazole and pentamidine, glucocorticoids may diminish the inflammatory response to severe pneumocystis infection and allow time for the antimicrobial agents to exert their effects. This theory is supported by the open lung biopsy findings in a patient with pneumocystis pneumonia who received high doses of methylprednisolone two days before biopsy. ${ }^{16}$ Instead of the intense interstitial mononuclear cell infiltrate usually seen in pneumocystis pneumonia there were only a few inflammatory cells in the interstitium. A further hypothesis is that phospholipase $A_{2}$ inhibition by glucocorticoids may retard the degradation of surfactant. ${ }^{18}$

In a study by MacFadden et al 10 patients with pneumocystis pneumonia and respiratory failure were given methylprednisolone in a dose of $40 \mathrm{mg}$ six hourly for seven days with their antimicrobial treatment; eight similar patients were given antimicrobial treatment alone. ${ }^{15}$ Nine of the 10 patients having methylprednisolone survived compared with two of the eight patients treated conventionally. Clinical improvement was evident within two 
Table 5 Methylprednisolone protocol

Infuse $1 \mathrm{~g}$ methylprednisolone intravenously in $250 \mathrm{ml}$ normal saline over one hour days 1-3 (no "tailing down" needed)

Ranitidine $150 \mathrm{mg}$ twice daily orally or $50 \mathrm{mg}$ thrice daily intravenously days $1-10$

Check serum potassium concentration before

methylprednisolone and daily days $2-4$

BM stix blood glucose estimation six hourly days 1-5

Daily arterial blood gas estimation

Transcutaneous oximeter for oxygen saturation

days of the start of corticosteroid treatment. None of the 10 patients showed clinical deterioration or recurrence of pneumocystis pneumonia on stopping methylprednisolone. Although infectious complications might be expected to occur more frequently during steroid treatment in patients with AIDS, this was not apparent during the study. In one patient, however, disseminated herpes zoster developed 48 hours after discontinuation of steroid treatment. Non-infectious complications were not observed.

Since this report appeared doubts have been cast on the efficacy of methylprednisolone. In a prospective double blind study of 41 patients with pneumocystis pneumonia and hypoxaemia (arterial oxygen tension $\left(\mathrm{PaO}_{2}\right)$ while they were breathing air $\leqslant 6.7 \mathrm{kPa}$ ) given intravenous methylprednisolone $(60 \mathrm{mg}$ four times daily, reduced over eight days) or placebo, no beneficial effects were shown in the treatmen group and there were no differences in survival. ${ }^{19}$ Our experiences with methylprednisolone are more encouraging. We have used much larger doses, $1 \mathrm{~g}$ daily in a pulse dose fashion for three days (table 5) in an open prospective study of 26 patients with pneumocystis pneumonia and variable hypoxaemia $\left(\mathrm{PaO}_{2} 4.3-12.9 \mathrm{kPa}\right.$ while they were breathing air) who were deteriorating despite conventional treatment. Twenty three patients responded and recovered fully; three patients died. No acute side effects were seen, but one patient became frankly jaundiced three weeks after the methylprednisolone treatment owing to reactivation of chronic hepatitis $\mathbf{B}$ infection. The reason for the better results in this study than in that of Clement et al ${ }^{19}$ may be the high dose of methylprednisolone. High dose methylprednisolone requires further evaluation in a prospective double blind clinical study.

\section{Fluid balance}

High dose intravenous co-trimoxazole is often diluted in 2 litres of normal saline a day and patients run the risk of fluid overload. Ill patients may be less able to excrete sodium and this may be compounded by the use of methylprednisolone. Severely hypoxaemic patients with pneumocystis pneumonia often have global ST segment and T wave changes indicating myocardial ischaemia on the electrocardiogram. Fluid overload in these patients may precipitate left ventricular failure. The oedema seen in transbronchial biopsy specimens may be a reflection of raised pulmonary venous pressure, and not just increased alveolar-capillary permeability. ${ }^{20}$ In these circumstances the logical approach is to try to reduce fluid overload and use intravenous diuretics.

Despite the "knee jerk" tendency to dilute the co-trimoxazole in a large volume of normal saline, the saline load can be reduced by using $5 \%$ dextrose or dextrose-saline and the volume of infusion can also be reduced considerably. The minimum volume needed to avoid precipitation of co-trimoxazole during infusion is $50-75 \mathrm{ml}$ of $5 \%$ dextrose for each $5 \mathrm{ml} \mathrm{am}$ poule of sterile co-trimoxazole solution (Septrin, Wellcome). Each $5 \mathrm{ml}$ ampoule contains $400 \mathrm{mg}$ of sulphamethoxazole and $80 \mathrm{mg}$ of trimethoprim. For a $60 \mathrm{~kg}$ patient prescribed $100 \mathrm{mg} / \mathrm{kg}$ sulphamethoxazole and $20 \mathrm{mg} / \mathrm{kg}$ trimethorpim a day (equivalent to $1.25 \mathrm{ml}$ strong sterile co-trimoxazole solution per $\mathrm{kg}$ body weight) $75 \mathrm{ml}$ ( 15 ampoules) of solution is required. The minimum volume of dilution is $750 \mathrm{ml}$, to be infused as $375 \mathrm{ml}$ twice daily.

Our experience suggests that intravenous frusemide $60-120 \mathrm{mg}$ is of temporary benefit (as measured by subjective reduction in dyspnoea, reduction in respiratory rate, and improvement in arterial oxygen tension) in patients with rapidly worsening arterial hypoxaemia. This temporary respite may be of value, pending decisions about instituting continuous positive airways pressure ventilation or transferring the patient to the intensive care unit for intubation and assisted ventilation (see below).

Continuous positive airways pressure ventilation In an attempt to avoid intubation and mechanical ventilation (see below) continuous positive airways pressure (CPAP) has been used in spontaneously breathing patients with pneumocystis pneumonia who remain hypoxaemic despite supplemental oxygen via a face mask. CPAP provides positive (end) expiratory pressure (PEEP) without the need for intubation. CPAP may be supplied by a nasal mask, as used for sleep apnoea, ${ }^{21}$ or by a tight fitting face mask. $^{22}$ The mechanism whereby CPAP improves ventilation is uncertain, but it may produce a "pneumatic splint," holding narrow, highly compliant airways open throughout the respiratory cycle. ${ }^{23} \mathrm{CPAP}$, like PEEP, increases functional residual capacity but it does not appear to affect cardiac output adversely. Older high flow CPAP systems used bag reservoirs in the circuit. This meant that whereas the positive pressure was maintained in expiration there were falls in pressure during peak inspiration and the patient's work of breathing was substantial. Newer CPAP circuits, which incorporate a flow generator (Medic-Aid, Pagham), do not produce a large pressure fall during inspiration and the work of breathing is reduced consequently. Complications of CPAP include pulmonary barotrauma, and when the face mask system of delivery has been used gastric aspiration and even facial pressure necrosis have been reported.

The short term effects of nasal CPAP $(10 \mathrm{~cm}$ $\mathrm{H}_{2} \mathrm{O}$ ) were assessed in eight patients with pneumocystis pneumonia who had mild to 
moderate hypoxaemia. ${ }^{24}$ Seven tolerated this with no complications but one patient did not. Mean $\mathrm{PaO}_{2}$ (during the breathing of room air) was $7.5 \mathrm{kPa}$ before and $9 \cdot 1 \mathrm{kPa}$ after 20 minutes of nasal CPAP, and the mean alveolar-arterial oxygen difference fell from 6.4 to $4.5 \mathrm{kPa}$. This study did not assess the value of CPAP in severely hypoxaemic patients or its clinical effectiveness in terms of outcome. We have given CPAP delivered via a face mask as an alternative to intubation and assisted ventilation to eight patients with pneumocystis pneumonia who developed respiratory failure refractory to supplemental oxygen via a face mask $\left(\mathrm{PaO}_{2} 4 \cdot 7-10 \cdot 4\right.$ (mean $\left.7 \cdot 1\right) \mathrm{kPa}$ during the breathing of $60 \%$ oxygen via a Ventimask). Seven patients were receiving intravenous cotrimoxazole and one intravenous pentamidine. CPAP (fractional inspired oxygen 0.6 and PEEP $5 \mathrm{~cm} \mathrm{H} \mathrm{H}_{2} \mathrm{O}$, apart from one patient $(10 \mathrm{~cm}$ $\left.\mathrm{H}_{2} \mathrm{O}\right)$ ) led to an improvement in arterial oxygenation in seven patients (mean $\mathrm{PaO}_{2} 10.4$ $\mathrm{kPa}$ four hours after the start of CPAP), and all were weaned off CPAP after four days on average and subsequently recovered. One patient died one hour after starting CPAP with deteriorating oxygenation, hypercapnia, and acute right heart failure. No major complications were seen, though three patients found the mask claustrophobic initially (but persisted with the treatment). We gave diamorphine $2.5 \mathrm{mg}$ subcutaneously as required to patients with persisting dyspnoea or claustrophobia if their arterial carbon dioxide tension was below $5.4 \mathrm{kPa}$. This protocol is summarised in table 6 .

\section{Assisted ventilation}

In the early 1980s the appropriateness of admission to the intensive care unit for intubation and assisted ventilation was rarely questioned by patients with AIDS who developed severe respiratory failure due to pneumocystis pneumonia. Because both their short and their long term prognosis was unknown, patients were frequently offered and generally accepted intensive care. ${ }^{3}$ By the mid 1980s in North America patients with AIDS and severe respiratory failure were admitted to the intensive care unit less often as their poor outcome became apparent. Only $14 \%$ of such patients admitted to San Francisco General Hospital from 1981 to 1985 survived, a proportion similar to that of other centres in North America. ${ }^{25-27}$ Admissions to the intensive care unit of patients with AIDS, pneumocystis

Table 6 Protocol for continuous positive airways pressure (CPAP)

\footnotetext{
Use a flow generator circuit (not a bag reservoir circuit)

Use tight fitting face mask or nasal mask

Begin with $5 \mathrm{~cm} \mathrm{H} \mathrm{C}_{2} \mathrm{O}$ PEEP and $\mathrm{FiO}_{2}=0.6$

Monitor arterial blood gases with an arterial line or ranscutaneous oximete

Chest radiographs during CPAP daily and at any stage if sudden increase in dyspnoea (to exclude a pneumothorax)

Treat persistent dyspnoea or claustrophobia from mask with cautious use of subcutaneous diamorphine $2.5 \mathrm{mg}$ as needed cautious use of subcutane
(only if $\mathrm{PaCO}_{2} \leqslant 5 \cdot 3 \mathrm{kPa}$ )
}

PEEP_-positive end expiratory pressure; $\mathrm{FiO}_{2}$ - fractional inspired oxygen; $\mathrm{PaCO}_{2}$-arterial carbon dioxide tension. pneumonia, and severe hypoxaemia had also declined over these years despite a continued increase in the total number of patients with AIDS and pneumocystis pneumonia admitted to other wards in the hospital. ${ }^{3}$ The major reason for this change was increased awareness by both patients and physicians of the probable outcome of admission to the intensive care unit and institution of assisted ventilation. ${ }^{28} 29$

The prognosis for patients with pneumocystis pneumonia and severe respiratory failure seems to have changed in North America. Hospital survival rates of $36-50 \%$ have been reported recently in such patients, ${ }^{3031}$ and a $40 \%$ survival following intubation and assisted ventilation was seen at San Francisco General Hospital in patients admitted from 1986 to $1988 .^{32}$ Although the reason for this improvement is not clear it is in part responsible for the fourfold increase in the use of intensive care facilities for patients with AIDS at San Francisco General Hospital over the past two years.

Our combined experience in London of 18 patients with AIDS and pneumocystis pneumonia requiring assisted ventilation shows a mortality rate in the short term of over $93 \%$ and there have been no long term $(>12$ months) survivors. In one of the recent studies in North America seven of the eight patients with moderately severe pneumocystis pneumonia who required assisted ventilation and survived were ventilated for only a short period after acute deterioration following bronchoscopy. ${ }^{33}$ Their better outcome probably reflects their moderately severe disease with good recovery from an acute deterioration rather than improved outcome from severe pneumocystis pneumonia. The improved prognosis may also be due to improved salvage therapy such as eflornithine or adjuvant treatment with methylprednisolone (or both).

The recent increase in admissions to intensive care units in North America is likely to be seen in Europe in the future because of the continued increase in the number of patients with AIDS, because AIDS is increasingly being seen as a chronic manageable condition, and because patients may live longer, in part as a result of early prophylaxis with drugs such as zidovudine and nebulised pentamidine. So, although the one year survival for pneumocystis pneumonia severe enough to require intubation and assisted ventilation remains very poor, ${ }^{32}$ indicating that in fact the prognosis for this subset of patients has not improved, more patients (and their physicians) are likely to want admission to the intensive care unit because of the perceived overall improvement in the prognosis of AIDS. When short term survival is unlikely or long term prognosis is poor, patients and their doctors may question the appropriateness of transfer to the intensive care unit for intubation and assisted ventilation. ${ }^{34}$ But we should not forget that patients with pneumocystis pneumonia and respiratory failure appear to have a better short term prognosis than similar patients with haematological malignancies or bone marrow transplants who are routinely offered admission to the intensive care unit for assisted ventilation. ${ }^{35}$ 
Table 7 Factors to be considered that favour instituting mechanical ventilation in HIV positive patients with pneumonia

First episode of pneumocystis pneumonia

Post-procedure deterioration-for example, after fibreoptic bronchoscopy and bronchoalveolar lavage

Rapidly deteriorating undiagnosed pneumonia in known HIV positive patients (or patients from "high risk" group)

Rapid deterioration despite high dose intravenous cotrimoxazole

Failed trial of CPAP

$\mathrm{PaO}_{2} \leqslant 7 \cdot 0 \mathrm{kPa}$ despite $\mathrm{FiO}_{2}=1$ with tight fitting face mask

Patient's wishes

Relative's wishes

Medical and nursing staff wishes

$\mathrm{PaO}_{2}$-arterial oxygen tension; other abbreviations as in table 6 .

In view of all this there are certain circumstances in which assisted ventilation will need to be considered by physicians or requested by patients. Possible criteria to be used when mechanical ventilation is being considered for HIV positive patients with pneumonia are shown in table 7 .

\section{Problems of HIV positive patients in the intensive care unit}

Caring for HIV positive patients in the intensive care unit may pose several difficulties (table 8). Problems that may be encountered may include:

1 Identifying or "flagging" the patient as being $H I V$ positive This may provoke anxiety in medical and nursing colleagues and requires considerable tact in discussions over management of the patient. Patient confidentiality is also particularly vulnerable in this environment. In some centres being HIV positive or "high risk" means that access is denied to the use of automated blood gas and electrolyte analysis in the intensive care unit and samples of blood may have to be transferred to a designated "high risk" laboratory, with consequent delays in analysis.

2 Cubicle versus open ward Ideally any HIV positive patient who is ventilated should be nursed in a cubicle. Full protective clothing should be worn (that is, goggles or visor, mask, cardiac surgeon's type hat, disposable nonabsorbable gown, and two pairs of surgeon's gloves) when "suctioning" (aspiration) is performed via the endotracheal tube. This is because the technique of suctioning aspirates bronchial secretions capable of transmitting HIV, and even with a low pressure technique minor suction catheter trauma will cause blood to mingle with the secretions. These secretions

Table 8 Problems of mechanically ventilated HIV positive patients in the intensive care unit

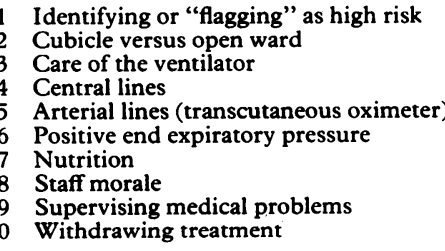

are aerosolised during suction and may be inhaled or absorbed on to mucosal surfaces of nursing staff or physiotherapists performing suctioning.

3 Care of the ventilator A separate article in this series ${ }^{36}$ has discussed control of infection policies. Where possible ventilators should be used with Pall's filters placed in the exhalation limb of the ventilator tubing. After use the ventilator tubing should be cleaned with $2 \%$ glutaraldehyde solution and the ventilator should be dismantled and cleaned according to local control of infection policies.

4 Central lines If these are thought necessary-for example, for infusing intravenous sedatives-they should be inserted by experienced staff using only the Seldinger technique. The internal jugular route is preferred in patients receiving positive pressure ventilation to reduce the risk of pneumothorax, which may be fatal in such patients. In a patient who has already sustained a pneumothorax (whether breathing spontaneously or during assisted ventilation) and has a chest drain in position the central line should be placed in the internal jugular vein on the same side as the pneumothorax to avoid the risk of damage to the other lung. "Traffic light" connectors should not be used as they may become disconnected and precipitate blood spillage.

5 Arterial lines An indwelling radial or femoral arterial line can provide on line blood pressure data and ready access to arterial blood for blood gas analysis. It also avoids repeated arterial puncture with its attendant risks of needlestick injury. Meticulous care must be taken with the arterial line, however, to prevent it dislodging or becoming disconnected. Some units prefer to perform intermittent blood gas analysis from an arterial puncture and use a transcutaneous oximeter to monitor oxygen saturation.

6 Positive end expiratory pressure In patients receiving positive pressure ventilation and requiring a high inspired oxygen concentration to maintain oxygenation, instituting PEEP may help to reduce the required oxygen concentration. Great care should be taken when PEEP is used in patients with pneumocystis pneumonia, however, as the incidence of spontaneous pneumothorax is high in these patients and appears to be higher in those patients with pneumocystis pneumonia who have assisted ventilation with PEEP than in HIV negative patients with other pulmonary problems (for example, bacterial pneumonia or the adult respiratory distress syndrome) who receive PEEP. Instituting PEEP may also reduce cardiac output and inotropic support may be necessary. In these circumstances inserting a Swan Ganz catheter (via the internal jugular route) may aid rational use of treatment. ${ }^{37}$

7 Nutrition Patients with pneumocystis pneumonia who are receiving assisted ventilation, like any patient with pneumonia, will require adequate nutrition. This may be given enterally or parenterally, but is probably better given enterally with a fine bore feeding tube as this avoids the need for a separate, dedicated 
central line. It has been recently suggested that enteral feeds with high fat and low carbohydrate formula may reduce the period for which assisted ventilation is required, ${ }^{38}$ but this require further evaluation in HIV positive patients with pneumocystis pneumonia who are receiving assisted ventilation.

8 Medical and nursing staff morale Staff morale will closely mirror the patient's condition. A high profile senior medical presence is a tremendous support to intensive care unit staff and frequent realistic discussions about treatment goals and likely prognosis are of paramount importance. Colleagues from other specialties may resent the use of intensive care unit beds by patients they regard as "no hopers."

9 Supervening medical problems HIV positive patients with pneumocystis pneumonia who are ventilated for long periods are at risk of developing other medical problems. The all too familiar scenario is progressive increases in $\mathrm{FiO}_{2}$ to maintain arterial $\mathrm{PaO}_{2}$, rising levels of PEEP to maintain oxygenation, multiple pneumothoraces requiring chest drains, subcutaneous emphysema, increasing inotropic support, secondary Gram negative pneumonia or septicaemia or both, renal failure, and cardiac failure.

10 Withdrawing treatment Difficult decisions about treatment arise-for example, about whether expensive intravenous broad spectrum antibiotics should be used or whether haemofiltration or haemodiafiltration should be started in sick patients with multiple organ failure who are unresponsive to treatment for their primary illness (pneumocystis pneumonia) and who have a very poor short and long term prognosis. We cannot be dogmatic about these issues and each case should be assessed individually. Decisions about continuing or withdrawing active treatment should be fully discussed, not only with the medical and nursing staff on the intensive care unit but also where possible with the patients and their partners or next of kin.

\section{Conclusions}

Most patients with pneumocystis pneumonia will respond to appropriate antimicrobial treatment. A few will deteriorate despite treatment; for those receiving nebulised pentamidine the change to intravenous co-trimoxazole and supplemental oxygen may be all that is necessary to secure recovery. For those deteriorating despite intravenous co-trimoxazole salvage treatment with trimetrexate or eflornithine may be considered. Adjuvant treatment with methylprednisolone in high dose may improve outcome in severely hypoxaemic patients, but further evaluation in prospective, double blind clinical studies is needed. Continuous positive airways pressure ventilation via a nasal or face mask appears to improve oxygenation in severely hypoxaemic patients with pneumocystis pneumonia and may obviate the need for intubation and positive pressure ventilation. In patients who receive assisted ventilation mortality remains high. In the intensive care unit, where possible, patients should be nursed in a cubicle and full protective clothing should be worn when "high risk" procedures are being carried out. If central lines are needed they should be placed by experienced staff using the internal jugular route. Frequent realistic discussions about therapeutic options, goals of treatment, and prognosis are essential to maintain staff morale. Where possible not only medical and nursing staff but also patients and their next of kin should take part in these discussions.

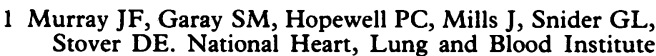
workshop summary: pulmonary complications of the acquired immunodeficiency syndrome: an update. $A m$ Rev Respir Dis 1987;135:504-9.

2 Rosen MJ, Tow TWY, Chuang MT, Teirstein AS. Prognosis of Pneumocystis carinii pneumonia in the acquired immunodeficiency syndrome [letter]. Ann Intern Med 1984;101:276.

3 Wachter RM, Luce JM, Turner J, Volberding P, Hopewel PC. Intensive care of patients with the acquired immunodeficiency syndrome; outcome and changing patterns of utilization. Am Rev Respir Dis 1986;134:891-6.

4 Kales CP, Murren JR, Torres RA, Crocco JA. Early predictors of in-hospital mortality for Pneumocystis carinit pneumonia in the acquired immunodeficiency syndrome. Ann Intern Med 1987;147:1413-7.

5 Brenner M, Ognibene FP, Lack EE, et al. Prognostic factors and life expectancy of patients with acquired immunodeficiency syndrome and Pneumocystis carinii pneumonia. Am Rev Respir Dis 1987;136:1199-206.

6 Sattler FR, Cowan R, Neilsen DM, Ruskin J. Trimethoprim-sulfamethoxazole compared with pentamidine for treatment of Pneumocystis carinii pneumonia in the treatment of Pneumocystis carinii pneumonia in the acquired immun

7 Miller RF, Godfrey-Faussett P, Semple SJG. Nebulised pentamidine as treatment for Pneumocystis carinii pneumonia in the acquired immunodeficiency syndrome. Thorax 1989;44:565-9.

8 Murray JF, Felton CP, Garay S, et al. Pulmonary complications of the acquired immunodeficiency syndrome: report of a National Heart, Lung and Blood Institute workshop. N Engl J Med 1984;310:1682-8.

9 Donelly H, Bernard EM, Rothkotter H, Gold JWM, Armstrong D. Distribution of pentamidine in patients Armstrong D. Distribution of pentamidin
with AIDS. J Infect Dis 1988;157:985-9.

10 Allegra CJ, Chabner BA, Tuazon CU, et al. Trimetrexate for the treatment of Pneumocystis carinii pneumonia in patients with the acquired immunodeficiency syndrome. N Engl J Med 1987;317:978-85

1 Golden JA, Sjoerdsma A, Santi DV. Pneumocystis carinii pneumonia treated with $\alpha$-difluoromethylornithine. West J Med 1984;141:613-23.

2 McLees BD, Barlow JLR, Kuzma RJ, Baringtang DC, Schecter PJ. Studies on successful eflornithine treatment of Pneumocystis carinii pneumonia (PCP) in AIDS patients failing conventional therapy. In: Third International Congress on AIDS. Washington, 1987:abstract TH.4.2:155.

13 Gazzard BG. Pneumocystis carinii pneumonia and its treatment in patients with AIDS. J Antimicrob Chemother ment in patients with
$1989 ; 23$ (suppl A):67-75.

14 Foltzer MA, Hannan SE, Kozak AJ. Pneumocystis pneumonia: response to corticosteroids [letter]. JAMA 1985;253:979.

5 MacFadden DK, Edelson JD, Hyland RH, Rodriguez $\mathrm{CH}$, Inouye $\mathrm{T}$, Rebuck AS. Corticosteroids as adjunctive therapy in treatment of Pneumocystis carinii pneumonia in patients with acquired immunodeficiency syndrome. Lancet 1987; ;:1477-9.

16 El-Sadr W; Sidhu G, Diamond G, et al. High dose corticosteroids as adjunctive therapy in severe Pneumocystis carinii pneumonia. AIDS Res 1986;2:349-55.

17 Fitzgerald W, Bevelaqua FA, Garay SM, et al. The role of open lung biopsy in patients with the acquired immunoopen lung biopsy in patients with the acquired
deficiency syndrome. Chest 1987;91:659-61.

18 Kernbaum S, Masliah J, Alandor LG, Bouton C, Christol D. Phospholypase activity of bronchoalveolar lavage fluid in rat Pneumocystis carinii pneumonia. Br J Exp Pathol 1983; 64:75-80.

19 Clement M, Edison R, Turner J, Montgomery B, Luce J, Feigal D, Hopewell P. Corticosteroids as adjunctive therapy in severe Pneumocystis carinii pneumonia. A prospective placebo-controlled trial [abstract]. Am Rev Respir Dis 1989;139(4):A250.

20 Yoneda K, Wuther PD. Experimental Pneumocystis carinii pneumonia in the rat: mechanism of alveolar injury. $\mathrm{Br} J$ Exp Pathol 1981;62:339-46.

21 Kerby GR, Mayer LS, Pingleton SK. Nocturnal positive pressure ventilation via nasal mask. Am Rev Respir Dis

22 Smith RA, Kirby RR, Gooding JM, Civetta JA. Continuous positive airway pressure (CPAP) by face mask. Crit Care (483 5 .

23 Sullivan CE, Issa FG, Berthon-Jones M, Eves L. Reversal of 
obstructive sleep apnoea by continuous positive pressure applied through the nares. Lancet 1981;i:862-5.

24 Kesten S, Rebuek AS. Nasal continuous positive airway pressure in Pneumocystis carinii pneumonia. Lancet pressure in $P$.

25 Stover DE, White DA, Romano PA, Gellene RA, Robeson WA. Spectrum of pulmonary diseases associated with the
acquired immunodeficiency syndrome. $A m J$ Med 1985; acquired im

26 Schein RMH, Fischl MA, Pitchenik AE, Sprung CL. ICU survival of patients with the acquired immunodeficiency syndrome. Crit Care Med 1986;14:1026-7.

27 Rosen MJ, Cueco RA, Teirstein AS. Outcome of intensive care in patients with the acquired immunodeficiency syndrome. J Inten Care Med 1986;1:55-60

28 Steinbrook R Lo B, Tirpack J, Dilley JW, Volberding PA. Ethical dilemmas in caring for patients with the acquired immunodeficiency syndrome. Ann Intern Med 1985;103: 787-90.

29 Wachter RM, Cooke M, Hopewell PC, Luce JM. Attitudes of medical residents regarding intensive care for patients
with the acquired immunodeficiency syndrome. Arch Intern Med 1988;148:149-52.

30 El-Sadr W, Simberkoff MS. Survival and prognostic factors in severe Pneumocystis carinii pneumonia requiring mechanical ventilation. Am Rev Respir Dis 1988; 137:1264-7.

31 Friedman Y, Franklin C, Rackow EC, Weil MH. Improved survival in patients with AIDS, Pneumocystis carini pneumonia and severe respiratory failure. Chest 1989;
96:862-6

32 Wachter RM, Russi MB, Hopewell PC, Luce JM. The improving survival rate after intensive care for Pneumocystis carinii pneumonia and respiratory failure [abstract]. Fifth International Conference on AIDS. Montreal, 1989:127.

33 Luce JM, Wachter RM, Hopewell PC. Intensive care of patients with the acquired immunodeficiency syndrome: time for a reassessment? Am Rev Respir Dis 1988; 137:1261-3.

34 Wachter RM, Luce JM, Hearst N, Lo B. Decisions about resuscitation: inequities among patients with different diseases but similar prognoses. Ann Intern Med 1989; 111:525-32.

35 Crawford SW, Schwartz DA, Peterson FB, Clark JG. Mechanical ventilation after marrow transplantation: risk factors and clinical outcome. Am Rev Respir Dis 1988; 137:682-7.

36 Hanson PJV, Collins JV. AIDS, aprons and elbow grease: preventing the nosocomial spread of human immuno-
deficiency virus and associated organisms. Thorax 1989; deficiency

37 Maxfield RA, Sorkin IB, Fazzini EP, Rapoport DM, Stenson WM, Goldring RM. Respiratory failure in patients with acquired immunodeficiency syndrome and Pneumocystis carinii pneumonia. Crit Care Med 1986; 14:443-9.

38 Al-Saady NM, Blackmore CM, Bennett ED. High fat, low carbohydrate enteral feeding lowers $\mathrm{PaCO}_{2}$ and reduces the period of ventilation in artificially ventilated patients. Inten Care Med 1989;15:290-5. 